\title{
Anti-phospholipid antibodies in the setting of thromboembolic events associated with severe COVID-19 pneumonia
}

\author{
Omaima Ibrahim Badr ${ }^{1,2}$, Wael Aly Elrefaey ${ }^{2}$, Mohammed Hassan Abu-Zaid ${ }^{3 *}$ (D) and Samah Hamdy Elmedany ${ }^{3,4}$
}

\begin{abstract}
Background: Thrombotic consequences have been reported in COVID-19-infected patients, especially those who are critically ill. Multiple studies have tested antiphospholipid antibodies (aPLs) among COVID-19 patients, but to date, the actual frequency of aPLs is still uncharted.

In this cohort study, we analyzed the outcomes of 173 consecutive patients with confirmed COVID-19 infection. Anti-phospholipid antibodies, which include anti-cardiolipin antibodies [aCL (IgM), aCL (IgG)], and B2-glycoprotein I antibodies [aß2GPI (IgM), a $32 \mathrm{GPI}(\mathrm{IgG})]$ were detected by using immunoassays. In contrast, lupus anti-coagulant (LAC) antibodies are identified through a coagulation-based assay.

Results: The study demonstrated a high incidence of thrombotic consequences in severe COVID pneumonia cases and supported an increased risk of developing aPLs following COVID-19 infection. Pulmonary embolism had the most common prevalence of all thrombotic events. Among the various aPLs tested in thrombotic patients, lupus anticoagulant (LAC) had the highest positivity (46.2\%). Most patients with arterial thromboembolism (stroke, myocardial infarction, limb ischemia, bowel ischemia, and renal artery thrombosis) had triple positivity of anti-phospholipid antibodies. Testing aPLs antibodies after 12 weeks of recovery for survived patients only 2 out of 23 patients had aPLs positivity compared to 35 out of 65 tested during hospital admission. Furthermore, we found no significant changes in aPLs positivity between survived and non-survived patients with thrombotic event.
\end{abstract}

Conclusions: aPLs increased transiently as an inflammatory-mediated condition. Individuals with aPLs triple positivity (positive $L A C, a C L$, and aB2GPI) had a considerable risk of arterial thromboembolism (ATE).

Keywords: COVID-19, Severe pneumonia, Venous thromboembolism, Arterial thromboembolism, Anti-phospholipid antibodies

\section{Background}

The coronavirus disease 2019 (COVID-19) caused by the severe acute respiratory syndrome coronavirus 2(SARS$\mathrm{CoV}-2)$ is a major public health emergency in recent times [1]. Multi-organ failure secondary to infection by coronavirus has been labeled as the primary reason for mortality in COVID-19 [2, 3].

\footnotetext{
*Correspondence: drmhassan113@yahoo.com

${ }^{3}$ Department of Rheumatology and Rehabilitation, Faculty of Medicine,

Tanta University, El-Geish Street, Tanta, Gharbia, Egypt

Full list of author information is available at the end of the article
}

Recently, several trials have reported an exceptionally high prevalence of thrombotic events, suggesting that these complications may contribute to death $[4,5]$. A variety of studies have revealed thromboembolic consequences, including venous thromboembolism (VTE) (pulmonary embolism (PE), and deep vein thrombosis (DVT)), as well as arterial thromboembolism (ATE), which includes (cerebral infarction, myocardial infarction, and limb arterial thrombosis). An autopsy of a COVID-19 victim revealed micro pulmonary thrombosis at a rate of up to $80 \%$ [6]. 
The crucial role of thrombo-inflammation and endothelial damage in thromboembolism has long been known [7]. The overproduction of IL-1, interleukin (IL-6), IL-8, and tumor necrosis factor (TNF), as a pro-inflammatory cytokine, is thought to be the cause of "cytokine release syndrome" or "cytokine storm." In addition to pro-inflammatory marker increases, hypercoagulability has been recognized as a key factor in determining the prognosis of those patients [8]; however, the actual mechanism for thromboembolic complications is still unknown.

Anti-phospholipid antibodies (aPLs) are a class of antibodies that include the anti-cardiolipin $(\mathrm{aCL})$, lupus anti-coagulant (LAC), and anti-B2-glycoprotein I (aB2GPI) antibodies, all of which have phospholipid-binding proteins as their principal targets. The relationship of viral infections with aPLs is described before in the literature. Individuals infected with viruses such as HIV, HCV, HBV, human T-lymphotropic virus type 1 (HTLV-1), Epstein-Barr virus (EBV), varicella virus, cytomegalovirus (CMV), parvovirus B19, streptococcal and staphylococcal infections, and gram-negative organisms are highly associated with aPLs positivity [9]. It postulates that by molecular mimicry, some of the infectious agents might induce nonpathogenic aPLs and pathogenic anti- $\beta 2$-GPI [9].

Reports of appearing aPLs in COVID-19 patients and their putative relationship to thrombosis have started to emerge in case series and case reports $[10,11]$. Anti-cardiolipin (aCL) and anti-B2-glycoprotein I (aB2GPI) antibodies were found in three critical COVID-19 patients having multiple cerebral infarctions in a previous study suggesting for the first time that COVID-19-related coagulopathy could be an acquired thrombophilia close to the spectrum of anti-phospholipid syndrome (APS) [11]. The major difference between APS and COVID19-associated thrombosis is the normal fibrinogen levels in APS, which comes in contrast to COVID-19 thrombosis [11].

Multiple studies have tested aPLs antibodies among COVID-19 patients [11-14], but to date, the real frequency of aPLs is still uncharted. This study aimed to shed light on the association of aPLs and the development of thromboembolic events (arterial and venous) in severe COVID-19 pneumonia patients and whether these positive antibodies continue after improvement, as there is minimal evidence in the previous investigations. We also seek to identify the type of aPLs found in patients with COVID-19, as well as the possible association of these aPLs with other distinctive characteristics of COVID-19.

\section{Methods}

\section{Study design}

A prospective cohort study was conducted at Al-Noor Specialist, tertiary care institute, Makkah, Saudi Arabia.

\section{Inclusion and exclusion criteria}

Between 21 October 2020 and 30 March 2021, all patients with severe COVID-19 pneumonia (SARS-CoV2-infected individuals with $\mathrm{SpO} 293 \%$ on room air, $\mathrm{PaO} 2 /$ FiO2 ratio less than $300 \mathrm{mmHg}$, rate of respiration $>30$ breaths/minute, or pulmonary infiltrates $>50 \%$ ) [15] with probable thromboembolic complications were included in our study. Patients under 18 years and those with a history of the anti-phospholipid syndrome were excluded.

\section{Data collection and study procedures}

Data were obtained from medical files and electronic records using a distinctive medical record number (MRN). Demographic information of the patients (age, gender, nationality, and smoking history), as well as clinical symptoms (cough, fever, SOB, body aches, headache, nausea, vomiting, diarrhea, loss of taste, and loss of smell), comorbidities, and a chest radiograph, were gathered at the admission time to the hospital. Clinical indicators such as (respiration rate, heart rate, and oxygen saturation percent on room air, limb weakness, calf pain, and abdomen rigidity) were gathered at the time of suspicion of thromboembolic consequences.

Age, smoking history, obesity (defined as a BMI > 30), D-dimer level, length of hospital stays, comorbidities, disseminated intravascular coagulation (DIC) including CBC (WBC, platelets, and hemoglobin level) and coagulation parameters (PT, PTT, and INR) were all collected as part of the risk assessment for thromboembolism.

Suspected thromboembolic complications, either venous or arterial thromboembolism, were diagnosed in COVID-19 patients utilizing a Computed Tomography pulmonary angiogram (CTPA) to diagnose pulmonary embolism (PE). Doppler ultrasound for the diagnosis of vascular (arterial/venous) thrombosis, brain computed tomography (CT) to detect infarction, ECG and echocardiography to diagnose myocardial ischemia, and abdominal CT angiography for diagnosis of vascular thrombosis and mesenteric/bowel ischemia.

Anti-phospholipid antibodies, which include anti-cardiolipin antibodies [aCL (IgM), aCL (IgG)], and B2-glycoprotein I antibodies [a $\beta 2$ GPI (IgM), a $\beta 2$ GPI (IgG)] were detected by using immunoassays that measure reactivity to cardiolipin, a phospholipid, and b2-glycoprotein I, a phospholipid-binding protein, respectively. Lupus anticoagulant (LAC) antibodies were identified through a coagulation-based assay that demonstrates prolongation 
of a phospholipid-dependent clotting time. The results of at least one anti-phospholipid antibody test were reported as being positive. The term "mono positivity" refers to one of the three aPLs (LAC, aCL, or a2GPI), "double positivity" refers to two of the three aPLs, and "triple positivity" refers to all three aPLs. All patients were given prophylactic anti-coagulants (subcutaneous fractionated or unfractionated heparin) during their hospital stay, according to the hospital VTE policy. Serum aPLs antibodies were retested after 12 weeks from the first sample for the previously positive survived cases with thrombotic events. All retested patients were discharged as outpatients, based on the COVID-19 hospital discharge criteria (afebrile for at least $24 \mathrm{~h}$ without anti-pyretics, improved respiratory symptoms such as cough and shortness of breath, and two negative specimens collected $24 \mathrm{~h}$ apart), and they were all on therapeutic oral anti-coagulants. The final date of follow-up was 10 July 2021.

\section{Statistical analysis plan}

Statistical Package for Social Science (SPSS) version 22 was used in analyzing the collected information after it was recorded, coded, and tabulated using Windows on a personal computer. Patients' demographic parameters, clinical signs and symptoms, comorbidities, and radiological findings were described using descriptive statistics. Kolmogorov-Smirnov test for normality was used to assess the distribution of continuous variables. Normally distributed continuous data were described as mean $\pm \mathrm{SD}$, and for data that were not normally distributed, median (interquartile range (IQR)) was used. Qualitative data were described as percentages (frequencies). The Mann-Whitney $U$ test was used to compare the median value for non-normally distributed continuous variables. The independent sample $t$ test was used to compare the mean value for normally distributed. A chi-squared test/ Fisher test was used as appropriate to compare proportions for qualitative variables. Paired comparison of nominal data was done using McNemar test. A confidence interval of $95 \%(p<0.05)$ was applied to characterize the statistical significance of the results, and the level of significance was assigned as $5 \%$.

\section{Ethical part and confidentiality}

The Saudi Arabian Ministry of 131 Health's institutional ethics board approved this study (No. H-02-K-076-0920-386).

\section{Results}

Among 960 admitted COVID-19 patients confirmed by a real-time polymerase chain reaction (PCR), 173 patients with severe pneumonia screened for thromboembolic complications, of which 65 patients had proven thromboembolic events. For the 65 patient aPLs were tested during hospitalization, 19 (29.2\%) patients were mono positive, 7 (10.7\%) were double-positive, and 9 (13.8\%) had triple-positivity. Following re-testing for aPLs in 23 patients 12 weeks after the initial sample, only 2 patients tested positive for aPLs (one patient had single aPLs positivity and the other patient had three aPLs test positivity) (Fig. 1).

Venous thrombosis includes (PE, DVT, and PE with DVT) was detected in 53(30\%) out of 173 patients; however, arterial thrombosis includes (stroke, limb ischemia, MI, renal artery thrombosis, and bowel ischemia) was detected in 12 (6.9\%) (Table 1$)$.

The two groups showed comparable baseline demographic characteristics except for age and smoking which were significantly higher in patients with thrombosis $(p$ $<0.001)$. There was no statistically significant difference between the two groups in the terms of gender distribution, nationality, and reported comorbidities $(p>0.05)$. Signs, symptoms, and outcome measures were also comparable in both groups, except for chest pain which was significantly higher in patients complicated by thrombosis, for further information on the baseline characteristics refer to (Table 2).

Both groups were comparable in their vital sign measurements (respiratory rate, heart rate, oxygen saturation) and various blood test measurements (HGB, WBCs, INR, PT, PTT, and platelet count). However, D-dimer measures were significantly higher in patients with thrombotic complications.

There was no significant difference in CT parenchymal findings between both groups. Regarding ECHO findings, RV dilatation, and dysfunction were significantly higher in patients with thrombosis.

Except for aB2GPI (IgG), which was significantly higher in the group with thrombotic events, there was no significant difference between the two groups in the proportion of patients positive for aPLs (LAC, aCL, or a2GPI IgM) ( $p$ $>0.05$ ) (Table 3).

Pulmonary embolism had the most common prevalence of all thrombotic events, 48 patients out of 65(73.8\%). Among the various aPLs tested in thrombotic patients, lupus anti-coagulant (LAC) had the highest positivity (46.2\%). The presence of any circulating aCL (IgM) or aCL (IgG) has been found in 14 patients $(\sim 21.54 \%)$. The presence of any circulating a $\beta 2$ GPI (IgM) and aß2GPI (IgG) has been found in $16(\sim 24.62 \%)$ patients. Most patients with arterial thromboembolism (stroke, MI, limb ischemia, bowel ischemia, and renal artery thrombosis) had triple positivity of anti-phospholipid antibodies (Table 4). 


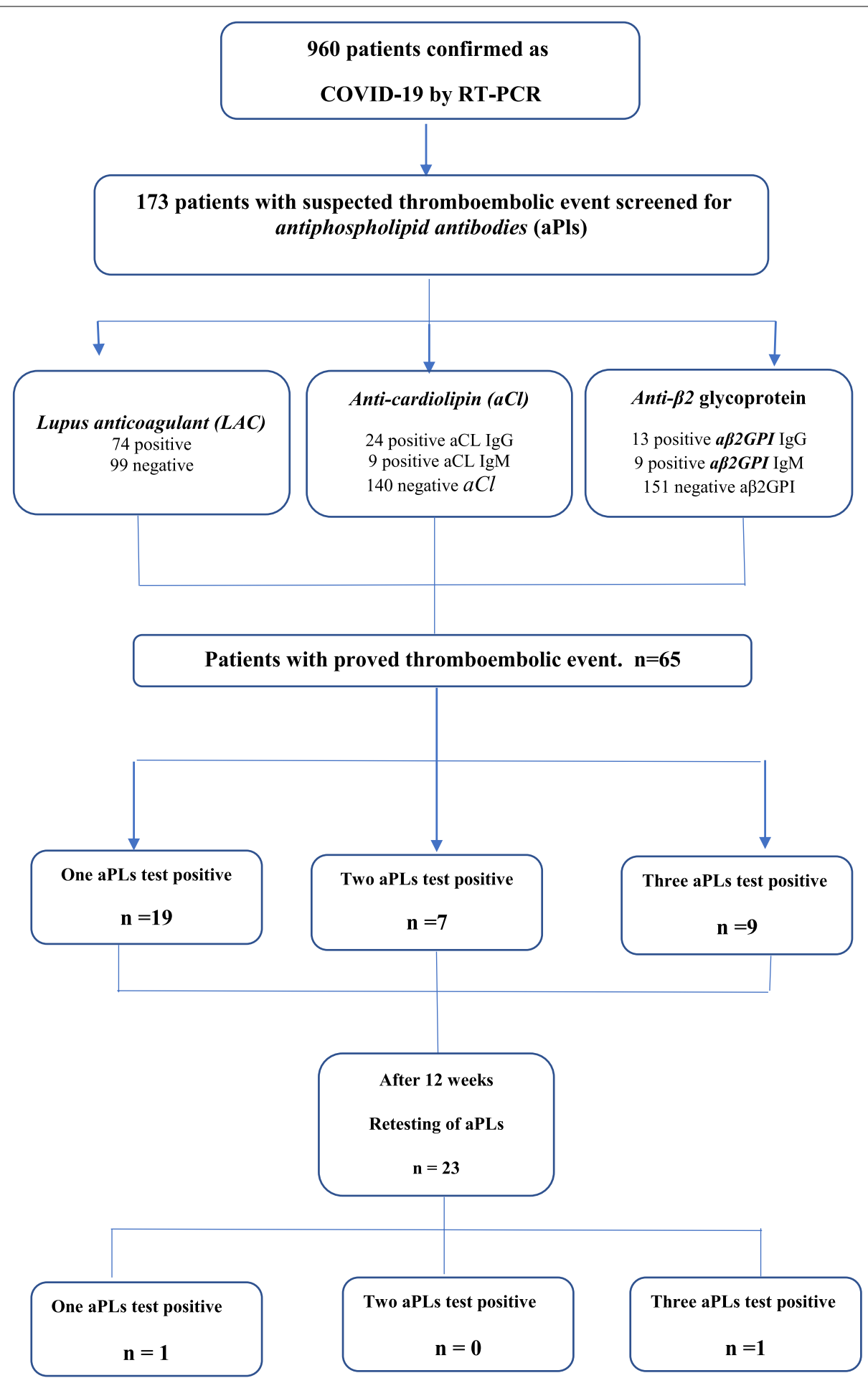

Fig. 1 Flow chart of the study design

In COVID-19 patients with thrombotic consequences, there was no statistically significant difference in the positivity of aPLs (LAC, aCL (IgG-IgM), or aß2GPI (IgG-IgM) between survivors and those who died (Table 5).
After 12 weeks, the number of positive LAC and aB2GPI IgM was significantly reduced $(p=0.005)$; however, there was no statistically significant difference between the two groups in aCL IgG and aCL IgM (Table 6). 
Table 1 Frequency and sort of the thromboembolic events associated with our COVID-19 patients

\begin{tabular}{ll}
\hline [DVT] Deep venous thromboembolism no. (\%) & $\mathbf{2 ( 1 . 2 )}$ \\
\hline [PE] Pulmonary embolism no. (\%) & $48(27.7)$ \\
PE + DVT no. (\%) & $3(1.7)$ \\
Renal artery thromboembolism no. (\%) & $1(0.5)$ \\
Stroke no. (\%) & $6(3.5)$ \\
Acute coronary syndrome no. (\%) & $2(1.2)$ \\
Mesenteric/bowel ischemia no. (\%) & $2(1.2)$ \\
Limb ischemia no. (\%) & $1(0.5)$ \\
No thrombosis no. (\%) & $108(62.4)$ \\
Total no. (\%) & $\mathbf{1 7 3 ( 1 0 0 )}$ \\
\hline
\end{tabular}

\section{Discussion}

Since the emergence of the COVID-19 pandemic, serious thrombotic consequences have been reported in infected patients, especially those who are critically ill [4]. Even with prophylactic or therapeutic anti-coagulation, COVID-19 patients experienced a higher-than-expected number of thrombotic episodes, both venous (pulmonary thromboembolism, venous sinus thrombosis, deep vein thrombosis) and arterial (myocardial infarction and stroke) [4].

In our present study, venous thrombosis includes (PE, DVT, and PE + DVT) was detected in 53 (30.6\%) out of 173 patients; however, arterial thrombosis includes (stroke, limb ischemia, MI, renal artery thrombosis, and bowel ischemia) was detected in 12 (6.9\%). This agreed with a recent meta-analysis [16] of 42 trials, including 8271 COVID-19 patients found an overall VTE incidence was $21 \%$, with a DVT rate of $20 \%$ and PE rate of $13 \%$, whereas the ATE rate was $2 \%$. In critically ill patients, the VTE rate was $31 \%$ and ATE rate was $5 \%$.

Our data revealed that pulmonary embolism was the most common thrombotic consequence, with PE occurring in 51 of $65(73.8 \%)$ patients who had thrombotic episodes. These findings are consistent with Klok et al. findings of a high incidence of VTE (31\%) leading to complications such as PE (80\%) [4]. In severe COVID19 pneumonia patients, the high prevalence of PE has been explained by the inflammatory nature of the disease rather than by an embolic mechanism of DVT [17].

Infections with COVID-19 may cause macrovascular and microvascular thrombosis through a variety of synergistic mechanisms. For example, a cytokine storm activates leukocytes, platelets, endothelium, hypoxic vaso-occlusion, and virus infection that directly activates immune and vascular cells [18]. COVID-19 is unique because it directly infects vascular endothelial cells; this dysfunction appears to be a critical signal for thrombosis [18]. Furthermore, the prevalence of thrombotic stroke, especially in young individuals, gives some clinical evidence that aPLs may be involved in endothelial dysfunction $[11,12]$. Our demographic characteristics agree with that except for the age; older age showed a significantly increased risk of developing thrombosis.

Like our results, multiple reports documented increased D-dimer levels $[8,19,20]$ associated with severe infection [21]. D-dimer levels seem a prognostic indicator as they increased to be 4-fold higher in patients who did not survive than survivors [8].

A recent study [14] demonstrated about one out of every two COVID-19 patients were tested positive for LAC, but aCL and a2GPI antibodies are less common (around 10\% for each). Among the various aPLs tested in our study, lupus anti-coagulant (LAC) had the highest rate of positivity (46.2\%) in the thrombotic group, whereas aCL (IgM or IgG) and anti-ß2 GPI (IgM or IgG) were about 21.6 and $24.6 \%$, respectively. This agreed with a recent meta-analysis involving 1159 patients (from 21 studies) admitted with COVID-19. The most frequent aPL detected was LAC, with pooled prevalence rate of $50.7 \%$ (95\% CI 34.8 to $66.5 \%$ ), whereas the prevalence rate of aCL (IgM or IgG) and anti- 32 GPI (IgM or IgG) were $13.9 \%$ (95\% CI 7.5 to $24.1 \%$ ) and $6.7 \%$ (95\% CI 3.5 to $12.5 \%)$, respectively [22].

The American Society of Hematology (ASH) performed an anti-phospholipid antibody (aPLs) testing. Only 4 out of 27 cases with COVID-19 exhibited lupus anti-coagulant (LAC) [23]. On the other hand, no patients tested positive for anti-aCL or anti-a2GPI antibodies. Nevertheless, the ASH strongly advised against routine aPLs testing in COVID-19 cases unless clinically recommended by the history or for a study protocol [24] due to the wellknown fact that aPLs might occur transiently after acute infection, inflammation, or thrombosis. Other ambivalent researchers have investigated the prothrombotic effects of aPLs and come up with conflicting conclusions. aPLs have been linked with the development of arterial thrombosis, notably pulmonary embolism and stroke, in several investigations [10, 25-29]. In conjunction with this research, this study suggests that COVID-19 infection increases the likelihood of acquiring aPLs. Although aPLs can alter hemostatic systems to cause thrombotic events, their existence in COVID-19 patients is not always accompanied by a thrombotic event.

A recent study has documented that aPLs, even in mild or transitory titers, are commonly present in hospitalized patients for COVID-19 [30]. Evidence in the literature has shown that patients with greater than one positive test, particularly those with triple positivity (LAC, aCL, and a $32 \mathrm{GPI}$ ), have an increased risk of thrombotic APS [30]. Double positivity (mostly LAC negative) is generally at lower thrombotic risk [31]. The 
Table 2 Baseline demographic, comorbidities, and associated diseases in two groups of COVID patients

\begin{tabular}{|c|c|c|c|}
\hline Demographic variable & $\begin{array}{l}\text { Patients with thrombotic events ( } n \\
=65)\end{array}$ & $\begin{array}{l}\text { Patients without thrombotic events ( } n \\
=108 \text { ) }\end{array}$ & $P$ value \\
\hline \multicolumn{4}{|l|}{ Demographics } \\
\hline Age (years) & $57.46(12.03)$ & $50.87(14.76)$ & $0.002^{* 1}$ \\
\hline \multicolumn{4}{|l|}{ Gender } \\
\hline $\begin{array}{l}\text { Male } \\
\text { Female }\end{array}$ & $\begin{array}{l}49(75.4) \\
16(24.6)\end{array}$ & $\begin{array}{l}71(65.7) \\
37(34.3)\end{array}$ & $0.183^{2}$ \\
\hline \multicolumn{4}{|l|}{ Nationality } \\
\hline $\begin{array}{l}\text { Saudi } \\
\text { Non-Saudi }\end{array}$ & $\begin{array}{l}26(40) \\
39(60)\end{array}$ & $\begin{array}{l}48(44.4) \\
60(55.6)\end{array}$ & $0.567^{2}$ \\
\hline BMI $\left(\mathrm{kg} / \mathrm{m}^{2}\right)$ & $27.36((23.5-31.2)$ & $26.02(24.04-31.23)$ & $0.944^{3}$ \\
\hline \multicolumn{4}{|l|}{ Smoking } \\
\hline No smoking & 23(35.4) & $64(59.3)$ & \\
\hline $\begin{array}{l}\text { Smoker } \\
\text { Unknown }\end{array}$ & $\begin{array}{l}39(60.0) \\
3(4.6)\end{array}$ & $\begin{array}{l}29(26.9) \\
15(13.9)\end{array}$ & $<0.001^{* 2}$ \\
\hline \multicolumn{4}{|l|}{ Comorbidities no. (\%) } \\
\hline Diabetes mellitus & $33(50.8)$ & 43(39.8) & $0.160^{2}$ \\
\hline Hypertension & $29(44.6)$ & $38(35.2)$ & $0.218^{2}$ \\
\hline Ischemic heart disease & $16(24.6)$ & $17(15.7)$ & $0.150^{2}$ \\
\hline Heart failure & $6(9.2)$ & $6(5.6)$ & $0.370^{2}$ \\
\hline Renal failure & $4(6.2)$ & $7(6.5)$ & $1.000^{2}$ \\
\hline Malignancy & $1(1.5)$ & 0 & NA \\
\hline Sickle cell disease & 0 & $3(2.8)$ & NA \\
\hline HIV & 0 & $1(0.9)$ & NA \\
\hline \multicolumn{4}{|c|}{ Pulmonary disease (other than pulmonary embolism) no. (\%) } \\
\hline No & $62(95.4)$ & $90(83.3)$ & \\
\hline COPD & $1(1.5)$ & $7(6.5)$ & \\
\hline $\begin{array}{l}\text { Asthma } \\
\text { Tuberculosis } \\
\text { IPF } \\
\text { Pulmonary HTN }\end{array}$ & $\begin{array}{l}1(1.5) \\
0 \\
0 \\
1(1.5)\end{array}$ & $\begin{array}{l}8(7.4) \\
2(1.9) \\
1(0.9) \\
0\end{array}$ & $0.110^{2}$ \\
\hline \multicolumn{4}{|c|}{ Sign and symptoms (at presentation to hospital) no. (\%) } \\
\hline Fever & $50(76.9)$ & $80(74.1)$ & $0.675^{2}$ \\
\hline Cough & $54(83.1)$ & $85(78.7)$ & $0.483^{2}$ \\
\hline Sore throat & 24(36.9) & $47(43.5)$ & $0.393^{2}$ \\
\hline Dyspnea & $58(89.2)$ & 98(90.7) & $0.747^{2}$ \\
\hline Hemoptysis & $7(10.8)$ & $11(10.2)$ & $0.903^{2}$ \\
\hline Chest pain & 25(38.5) & $26(24.1)$ & $0.044^{* 2}$ \\
\hline Vomiting & $12(18.5)$ & $28(25.9)$ & $0.259^{2}$ \\
\hline Diarrhea & $16(24.6)$ & 33(30.6) & $0.401^{2}$ \\
\hline Nausea & $22(33.8)$ & $39(36.1)$ & $0.763^{2}$ \\
\hline Loss of smell & $6(9.2)$ & $18(16.7)$ & $0.171^{2}$ \\
\hline Loss of taste & $8(12.3)$ & $17(15.7)$ & $0.534^{2}$ \\
\hline Headache & $28(43.1)$ & $42(38.9)$ & $0.587^{2}$ \\
\hline Bone ache & $37(56.9)$ & $64(59.3)$ & $0.763^{2}$ \\
\hline Limb weakness & $4(6.15)$ & 0 & NA \\
\hline Calf pain & $4(6.15)$ & 0 & NA \\
\hline Abdominal tenderness & $2(3.08)$ & 0 & NA \\
\hline \multicolumn{4}{|l|}{ Other no. (\%) } \\
\hline Duration of stay at hospital (days) & $16(10-23)$ & 14(10-20.75) & $0.468^{3}$ \\
\hline $\begin{array}{l}\text { Duration between admission and suspicion of } \\
\text { thrombosis }\end{array}$ & $7(3-10)$ & $5(3-9.75)$ & $0.509^{3}$ \\
\hline
\end{tabular}


Table 2 (continued)

\begin{tabular}{|c|c|c|c|}
\hline Demographic variable & $\begin{array}{l}\text { Patients with thrombotic events ( } n \\
=65 \text { ) }\end{array}$ & $\begin{array}{l}\text { Patients without thrombotic events ( } n \\
=108 \text { ) }\end{array}$ & $P$ value \\
\hline \multicolumn{4}{|l|}{ Outcomes (survival) no. (\%) } \\
\hline $\begin{array}{l}\text { Survived } \\
\text { Died }\end{array}$ & $\begin{array}{l}47(72.3) \\
18(27.7)\end{array}$ & $\begin{array}{l}74(68.5) \\
34(31.5)\end{array}$ & $0.599^{2}$ \\
\hline ICU admission (yes) & $51(78.5)$ & $80(74.1)$ & $0.515^{2}$ \\
\hline Intubation (yes) & $39(60.0)$ & $60(55.6)$ & $0.567^{2}$ \\
\hline Duration of stay at the ICU & $6(1-11)$ & $5.5(0-13)$ & $0.758^{3}$ \\
\hline
\end{tabular}

Values are the mean and SD, median (IQR) or $n$ (\%), BMI body mass index, $\mathrm{kg} / \mathrm{m}^{2}$ mean (SD), HIV human immunodeficiency virus, COPD chronic obstructive pulmonary disease, IPF idiopathic pulmonary fibrosis, HTN hypertension. ICU intensive care unit, NA not applicable

${ }^{1}$ Independent-sample $t$ test

${ }^{2}$ Chi-square test

${ }^{3}$ Mann-Whitney test

Table 3 Vital signs, blood tests, radiological finding, ECHO findings, and frequency of positive aPLs in both groups at the time of suspicion of thrombosis

\begin{tabular}{|c|c|c|c|}
\hline Variable & $\begin{array}{l}\text { Patients with thrombotic events } \\
(n=65)\end{array}$ & $\begin{array}{l}\text { Patients without thrombotic events } \\
(n=108)\end{array}$ & $P$ \\
\hline Respiratory rate & $30(26-34.5)$ & $29(25.25-32)$ & $0.429^{3}$ \\
\hline Heart rate $(b / m)$ & 110(100-122) & 109(100-117.8) & $0.285^{3}$ \\
\hline Oxygen saturation $\%$ & $88(80-90)$ & $88(82-90)$ & $0.834^{3}$ \\
\hline $\mathrm{HGB}(\mathrm{g} / \mathrm{L})$ & $124.48(22.2)$ & 123.23(23.53) & $0.731^{1}$ \\
\hline $\operatorname{WBCs}\left(10^{9} / \mathrm{L}\right)$ & 11.27(7.3-14.4) & $9.5(6.8-13.8)$ & $0.225^{3}$ \\
\hline Platelet count $\left(10^{9} / \mathrm{L}\right.$ & $253(187.5-336.5)$ & 256.5(194-339) & $0.909^{3}$ \\
\hline PTT $(s)$ & $34.8(29.6-39.35)$ & 34.3(30.7-38.8) & $0.614^{3}$ \\
\hline PT $(s)$ & $13.4(12.1-14.85)$ & 13.05(12.3-14.6) & $0.943^{3}$ \\
\hline INR & $1.14(0.2)$ & $1.11(0.2559)$ & $0.085^{1}$ \\
\hline D-dimer(mg/L) & 12.99(6.8-21.9) & $3.68(2.05-7.2)$ & $<0.001^{* 3}$ \\
\hline \multicolumn{4}{|l|}{ CT parenchymal findings no. (\%) } \\
\hline $\begin{array}{l}\text { No pulmonary infiltrate } \\
\text { Bilateral peripheral ground glass }\end{array}$ & $\begin{array}{l}2(3.1) \\
33(50.8)\end{array}$ & $\begin{array}{l}50(46.3 \\
50(46.3)\end{array}$ & \multirow[t]{3}{*}{$0.179^{2}$} \\
\hline Bilateral peripheral ground glass with consolidation & 28(43.1) & $8(7.4)$ & \\
\hline Unilateral peripheral ground glass & $2(3.1)$ & 0 & \\
\hline \multicolumn{4}{|l|}{ ECHO no. (\%) } \\
\hline Not done & 10(15.4) & $17(15.7)$ & \\
\hline Normal & 10(15.4) & 19(17.6) & \\
\hline $\begin{array}{l}\text { VRV dilatation or dysfunction } \\
\text { Left ventricular dysfunction } \\
\text { Pulmonary HTN }\end{array}$ & $\begin{array}{l}25(38.5) \\
6(9.2) \\
14(21.5)\end{array}$ & $\begin{array}{l}19(17.6) \\
23(21.3) \\
30(27.8)\end{array}$ & $0.018^{* 2}$ \\
\hline \multicolumn{4}{|l|}{ Positive aPLs no. (\%) } \\
\hline LAC & $30(46.2)$ & $44(40.7)$ & $0.486^{2}$ \\
\hline $\begin{array}{l}\mathrm{aCL} \lg G \\
\mathrm{aCL} \lg \mathrm{M}\end{array}$ & $\begin{array}{l}10(15.4) \\
4(6.2)\end{array}$ & $\begin{array}{l}14(13) \\
5(4.6)\end{array}$ & $\begin{array}{l}0.655^{2} \\
0.730^{2}\end{array}$ \\
\hline $\begin{array}{l}\text { aß2GPI lgG } \\
a \beta 2 G P I \lg M\end{array}$ & $\begin{array}{l}10(15.4) \\
6(9.2)\end{array}$ & $\begin{array}{l}3(2.8) \\
3(2.8)\end{array}$ & $\begin{array}{l}0.005^{* 2} \\
0.082^{2}\end{array}$ \\
\hline Any positive aPLs & $35(53.8 \%)$ & $53(49 \%)$ & $0.543^{2}$ \\
\hline
\end{tabular}

HGB hemoglobin, WBCs white blood cells, PTT partial thromboplastin time, PT prothrombin time, INR international normalized ratio. CT computerized tomography, $E C H O$ echocardiogram, HTN hypertension, aPLs anti-phospholipid antibodies, LAC lupus anti-coagulant, $a C L$ anti-cardiolipin, a $\beta 2 G P I$ anti- $\beta 2$ glycoprotein

${ }^{*} P<0.05$ (significant value)

${ }^{1}$ Independent-sample $t$ test

${ }^{2}$ Chi-square test

${ }^{3}$ Mann-Whitney test 
Table 4 aPLs in different types of thrombosis in COVID-19 patients $(n=65)$

\begin{tabular}{|c|c|c|c|c|c|c|c|c|}
\hline Positive aPLs & $\begin{array}{l}\text { DVT + PE } \\
3(4.6 \%)\end{array}$ & $\begin{array}{l}\mathrm{PE} \\
48(73.8 \%)\end{array}$ & $\begin{array}{l}\text { DVT } \\
2(3.1 \%)\end{array}$ & $\begin{array}{l}\text { Stroke } \\
6(9.2 \%)\end{array}$ & $\begin{array}{l}\text { Limb } \\
\text { ischemia } \\
1(1.5 \%)\end{array}$ & $\begin{array}{l}\text { MI } \\
2(3.1 \%)\end{array}$ & $\begin{array}{l}\text { RAT } \\
1(1.5 \%)\end{array}$ & $\begin{array}{l}\text { Bowel ischemia } \\
2(3.1 \%)\end{array}$ \\
\hline LAC & $1(33.3)$ & 16(33.3) & $2(100)$ & $5(83.3)$ & $1(100)$ & $2(100)$ & $1(100)$ & $2(100)$ \\
\hline $\begin{array}{l}\mathrm{aCL} \lg G \\
\mathrm{aCL} \lg M\end{array}$ & $\begin{array}{l}0(0) \\
0(0)\end{array}$ & $\begin{array}{l}5(10.4) \\
3(6.3)\end{array}$ & $\begin{array}{l}0(0) \\
0(0)\end{array}$ & $\begin{array}{l}2(33.3) \\
1(16.7)\end{array}$ & $\begin{array}{l}0(0) \\
0(0)\end{array}$ & $\begin{array}{l}1(50) \\
0(0)\end{array}$ & $\begin{array}{l}1(100) \\
0(0)\end{array}$ & $\begin{array}{l}1(50) \\
0(0)\end{array}$ \\
\hline $\begin{array}{l}\text { a } \beta 2 \mathrm{GPI} \text { gG } \\
\text { a } 32 \mathrm{GPI} \text { gM }\end{array}$ & $\begin{array}{l}0(0) \\
0(0)\end{array}$ & $6(12.5) 1(2.1)$ & $\begin{array}{l}1(50) \\
0(0)\end{array}$ & $\begin{array}{l}2(33.3) \\
2(33.3)\end{array}$ & $\begin{array}{l}0(0) \\
0(0)\end{array}$ & $\begin{array}{l}1(50) \\
1(50)\end{array}$ & $\begin{array}{l}1(100) \\
0(0)\end{array}$ & $\begin{array}{l}1(50) \\
0(0)\end{array}$ \\
\hline
\end{tabular}

$a P L s$ anti-phospholipid antibodies, $L A C$ lupus anti-coagulant, $a C L$ anti-cardiolipin, $a \beta 2 G P I$ anti- $\beta 2$ glycoprotein, $M I$ myocardial infarction, $R A T$ renal artery thrombosis, data are expressed as no. (\%)

Table 5 aPLs and survival in COVID-19 patients with thrombotic events

\begin{tabular}{llll}
\hline Positive aPLs & Survived $(\boldsymbol{n}=\mathbf{4 7})$ & $\operatorname{Died}(\boldsymbol{n}=\mathbf{1 8})$ & $\boldsymbol{P}$ \\
\hline LAC & $19(40.4)$ & $11(61.1)$ & $0.134^{2}$ \\
aCL IgG & $6(12.8)$ & $4(22.2)$ & $0.445^{2}$ \\
aCL IgM & $3(6.4)$ & $1(5.6)$ & $1.000^{2}$ \\
a 32 GPI lgG & $8(17.0)$ & $2(11.1)$ & $0.713^{2}$ \\
a 2 GPI lgM & $5(10.6)$ & $1(5.6)$ & $1.000^{2}$ \\
\hline
\end{tabular}

$a P L s$ anti-phospholipid antibodies, $L A C$ lupus anti-coagulant, $a C L$ anticardiolipin, aß2GPI anti- $\beta 2$ glycoprotein, data are expressed as no. (\%)

${ }^{2}$ Chi square test

Table 6 Comparing aPLs during hospital admission and after 12 weeks of the first sample in survived positive patients (any positive aPLs test) with thrombotic events $(N=23)$

\begin{tabular}{|c|c|c|c|}
\hline$a P L s$ & $\begin{array}{l}\text { During admission } \\
(n=23)\end{array}$ & $\begin{array}{l}\text { After } 12 \text { weeks } \\
(n=23)\end{array}$ & $P$ \\
\hline \multicolumn{4}{|l|}{ LAC } \\
\hline $\begin{array}{l}\text { Positive } \\
\text { Negative }\end{array}$ & $\begin{array}{l}19(82.6) \\
4(17.4)\end{array}$ & $\begin{array}{l}1(4.3) \\
22(95.7)\end{array}$ & $<0.001^{* 4}$ \\
\hline \multicolumn{4}{|l|}{ aß2GPI lgG } \\
\hline Positive & $8(34.8)$ & $1(4.3)$ & $0.016^{* 4}$ \\
\hline Negative & $15(65.2)$ & $22(95.7)$ & \\
\hline \multicolumn{4}{|l|}{ aß2GPI IgM } \\
\hline $\begin{array}{l}\text { Positive } \\
\text { Negative }\end{array}$ & $\begin{array}{l}5(21.7) \\
18(78.3)\end{array}$ & $\begin{array}{l}1(4.3) \\
22(95.7)\end{array}$ & $0.219^{4}$ \\
\hline \multicolumn{4}{|l|}{$\mathrm{aCL} \lg G$} \\
\hline Positive & $6(26.1)$ & $1(4.3)$ & $0.125^{4}$ \\
\hline Negative & 17(73.9) & $22(95.7)$ & \\
\hline \multicolumn{4}{|l|}{$\mathrm{aCL} \lg \mathrm{M}$} \\
\hline $\begin{array}{l}\text { Positive } \\
\text { Negative }\end{array}$ & $\begin{array}{l}3(13) \\
20(87)\end{array}$ & $\begin{array}{l}0(0) \\
23(100)\end{array}$ & $0.250^{4}$ \\
\hline
\end{tabular}

$a P L s$ anti-phospholipid antibodies, $L A C$ lupus anti-coagulant, $a C L$ anticardiolipin, $a \beta 2 \mathrm{GPI}$ anti- $\beta 2$ glycoprotein, NA not applicable, data are expressed as no. (\%)

${ }^{*} P<0.05$ (significant value)

${ }^{4}$ McNemar test reliability of the results was affected by the presence of antibodies of the same isotype [32]. Patients with isolated positive LAC, but negative $\mathrm{aCL}$ and $\mathrm{a} \beta 2 \mathrm{GPI}$, have a low risk of a thromboembolic event [33]. In agreement with that, $13.8 \%$ of our thrombotic patients had triple positivity of (LAC, anti-cardiolipin, and anti- $\beta 2$ glycoprotein I) antibodies. Also, we demonstrated that patients with arterial thrombosis (stroke, MI, limb ischemia, bowel ischemia, and renal artery thrombosis) had triple positivity of anti-phospholipid antibodies.

Although the presence of aPLs is characteristic of many infections, their occurrence does not always imply the development of thrombotic complications and, consequently, the anti-phospholipid syndrome (APS) [33]. The frequency of aPL antibodies involving a healthy population is demonstrated in studies with relatively low percentages, e.g., in a healthy control cohort of 200 people, IgG/IgM/IgA aCL $1 \% / 1 \% / 3 \%$, and $\operatorname{IgG} / \operatorname{IgM} /$ IgA anti- $\beta 2$ GPI $4 \% / 1 \% / 1 \%$ showed elevated levels [34]. Another study found that the prevalence of aPLs in the healthy population ranged from 1 to $5.6 \%$ [35]. In severe COVID-19, aPLs (aCL and a2GPI Ig) increase, but not in mild cases, suggesting that a vigorous anti-viral immunoglobulin response, potentially initiated in the bronchial mucosa, may cause systemic autoimmunity [26].

The Subcommittee for the Standardization (SCC) for LA and aPLs of the International Society of Thrombosis and Hemostasis (ISTH), in its latest update, endorses testing all three tests (LAC, aCL, and a2GPI) to detect APS-related thrombosis and should also validate positive laboratory findings 12 weeks following the original assessment [30]. Re-testing after 3 months is indicated to ensure reliability, especially in cases of an initial triple-positive test [30].

In our study, we retested aPLs for only 23 patients after 12 weeks from the initial sample and found that 2 out of 23 previously positive patients for aPLs were positive after 12 weeks. This finding is consistent with a previous study that found a reduction in aPLs positivity (9 patients out of 10 were negative) after 1 month follow-up [36], raising the fact that these aPLs increase 
transiently, as an inflammatory-mediated condition, and do not remain high enough to meet current APS classification requirements.

We found no significant differences in aPLs positivity between survived and dead patients, which is consistent with previous research [37], which found no significant link between aPLs positivity and mortality in COVID-19 patients with thrombotic complications. This could be explained by the associated severe pneumonia, which is the leading cause of death in COVID-19 patients.

Our study is limited by the small number of cases included (single-center study). Therefore, it is essential to conduct further studies that specifically test aPL antibodies in a larger context to make subsequent important statements about the role of APS in COVID-19 and further strengthen the significance of the described comparisons.

Also, the assessment of LAC in our study was challenged using unfractionated heparin and low molecular weight heparin (LWMH) that can lead to false-positive results. Anti-phospholipid antibodies (aCL and aß2GPI) detection is tiresome. There are several commercial assays, and even for the identical assays, inter-laboratory variability is considerable [38]. Furthermore, ELISA test results for aCL and 2GPI should be regarded positive if they are higher than the cut-off value, which is determined as more than the 99th percentile [39].

\section{Conclusions}

Our study revealed a high incidence of thrombotic consequences in severe COVID-19 pneumonia cases. This study supports an increased risk of developing aPLs following COVID-19 infection. Although aPLs can modify the hemostatic mechanisms towards thrombotic phenomena, their presence is not always accompanied by a thrombotic event in COVID-19 patients. These aPLs increased transiently as an inflammatory-mediated condition and did not remain high enough to meet current APS classification requirements. Individuals with aPLs triple positivity had a marked risk of arterial thrombosis. Also, we did not detect significant differences between survived and non-survived patients regarding the positivity of aPLs. Therefore, we did not support screening COVID-19 patients for aPL by evidence.

\footnotetext{
Abbreviations

COVID-19: Coronavirus disease 2019; SARS-CoV-2: Severe acute respiratory syndrome coronavirus 2; VTE: Venous thromboembolism; PE: Pulmonary embolism; DVT: Deep vein thrombosis; ATE: Arterial thrombosis; IL: Interleukin; TNF: Tumor necrosis factor; aPLs: Anti-phospholipid antibodies; aCL: Anti-cardiolipin; LAC: Lupus anti-coagulant; aB2GPI: Anti-B2-glycoprotein I; HTLV-1: Human T-lymphotropic virus type 1; EBV: Epstein-Barr virus; CMV: Cytomegalovirus; DIC: Disseminated intravascular coagulation; CTPA: Computed tomography pulmonary angiogram.
}

\section{Acknowledgements}

Not applicable

\section{Authors' contributions}

Conceptualization and design: Omaima Ibrahim Badr, Wael Aly Elrefaey, Mohammed Hassan Abu-Zaid and Samah Hamdy Elmedany. Acquisition of data: Omaima Ibrahim Badr and Wael Aly Elrefaey. Formal analysis: Samah Hamdy Elmedany and Omaima Ibrahim Badr. Investigation: Omaima Ibrahim Badr and Wael Aly Elrefaey. Methodology: Omaima Ibrahim Badr and Samah Hamdy Elmedany. Validation: Omaima Ibrahim Badr and Samah Hamdy Elmedany. Writing — original draft: Samah Hamdy Elmedany, Mohammed Hassan, AbuZaid, and Omaima Ibrahim Badr. Final approval of the version to be submitted: Omaima Ibrahim Badr, Wael Aly Elrefaey, Mohammed Hassan Abu-Zaid, and Samah Hamdy Elmedany. The authors read and approved the final manuscript.

\section{Funding}

The author(s) received no financial support for the research, authorship, and/ or publication of this article.

\section{Availability of data and materials}

The data will be available upon reasonable request.

\section{Declarations}

\section{Ethics approval and consent to participate}

The Saudi Arabian Ministry of 131 Health's institutional ethics board approved this study (No. H-02-K-076-0920-386). Informed written consents from all patients were obtained in accordance with the local ethical committee.

\section{Consent for publication}

Not applicable

\section{Competing interests}

The authors declare that they have no competing interests.

\section{Author details}

${ }^{1}$ Department of Chest Medicine, Faculty of Medicine, Mansoura University, Dakahlia Governorate, Egypt. ${ }^{2}$ Department of Chest Medicine, Al Noor Specialist Hospital, Mecca, Saudi Arabia. ${ }^{3}$ Department of Rheumatology and Rehabilitation, Faculty of Medicine, Tanta University, El-Geish Street, Tanta, Gharbia, Egypt. ${ }^{4}$ Department of Rheumatology and Rehabilitation Medicine, Al Noor Specialist Hospital, Mecca, Saudi Arabia.

Received: 26 October 2021 Accepted: 4 December 2021

Published online: 18 January 2022

\section{References}

1. Wang D, Hu B, Hu C, Zhu F, Liu X, Zhang J et al (2020) Clinical characteristics of 138 hospitalized patients with 2019 novel coronavirus-infected pneumonia in Wuhan, China. JAMA 323:1061-1069

2. Rodriguez-Morales AJ, Cardona-Ospina JA, Gutiérrez-Ocampo E, Villamizar-Peña R, Holguin-Rivera Y, Escalera-Antezana JP et al (2020) Clinical, laboratory and imaging features of COVID-19: a systematic review and metaanalysis. Travel Med Infect Dis 34:101623

3. Borges do Nascimento IJ, Cacic N, Abdulazeem HM, von Groote TC, Jayarajah U, Weerasekara I et al (2020) Novel coronavirus infection (COVID-19) in APS and coronavirus disease-19 thrombosis https:// humans: a scoping review and meta-analysis. J Clin Med 9:941

4. Klok FA, Kruip MJHA, van der Meer NJM, Arbous MS, Gommers DAMPJ, Kant KM et al (2020) Incidence of thrombotic complications in critically ill ICU patients with COVID-19. Thromb Res 191:145-147

5. Lodigiani C, lapichino G, Carenzo L, Cecconi M, Ferrazzi P, Sebastian T et al (2020) Venous and arterial thromboembolic complications in COVID-19 patients admitted to an academic hospital in Milan, Italy. Thromb Res 191:9-14

6. Dolhnikoff M, Duarte-Neto AN, de Almeida Monteiro RA, Ferraz da Silva LF, Pierre de Oliveira E, Saldiva PHN et al (2020) Pathological evidence 
of pulmonary thrombotic phenomena in severe COVID-19. J Thromb Haemost 18(6):1517-1519. https://doi.org/10.1111/jth.14844

7. Meduri GU, Kohler G, Headley S, Tolley E, Stentz F, Postlethwaite A (1995) Inflammatory cytokines in the BAL of patients with ARDS. Persistent elevation overtime predicts poor outcome. Chest. 108:1303-1314

8. Tang N, Li D, Wang X, Sun Z (2020) Abnormal coagulation parameters are associated with poor prognosis in patients with novel coronavirus pneumonia. J Thromb Haemost 18:844-847

9. Abdel-Wahab N, Talathi S, Lopez-Olivo MA, Suarez- Almazor ME (2018) Risk of developing antiphospholipid antibodies following viral infection: a systematic review and meta-analysis. Lupus 27:572-583

10. Devreese J, Linskens A, Benoit D, Peperstraete H (2020) Antiphospholipid antibodies in patients with COVID-19: A relevant observation? J Thromb Haemost 18:2191-2201

11. Zhang Y, Xiao M, Zhang S, Xia P, Cao W, Jiang W et al (2020) Coagulopathy and antiphospholipid antibodies in patients with Covid-19. N Engl J Med 382:e38

12. Bowles L, Platton S, Yartey N, Dave M, Lee K, Hart DP et al (2020) Lupus anticoagulant and abnormal coagulation tests in patients with Covid-19. N Engl J Med 383:288-290

13. Helms J, Tacquard C, Severac F, Leonard-Lorant I, Ohana M, Delabranche $X$ et al (2020) High risk of thrombosis in patients in severe SARS-CoV-2 infection: a multicenter prospective cohort study. Intensive Care Med 46:1089-1098

14. Harzallah I, Debliquis A, Drénou B (2020) Lupus anticoagulant is frequent in patients with Covid-19. J Thromb Haemost 18:2064-2065

15. National Institutes of Health. $\mathrm{NIH}$ covid-19 treatment guidelines. Clinical spectrum of SARS-CoV-2 Infection. 2020. https://www.Covid19treatmen tguidelines.nih.gov/overview/clinical/spectrum

16. Malas MB, Naazie IN, Elsayed N, Mathlouthi A, Marmor R, Clary B (2020) Thromboembolism risk of COVID-19 is high and associated with higher risk of mortality: a systematic review and meta-analysis. EClinicalMedicine 29:10639

17. Fox SE, Akmatbekov A, Harbert JL, Li G, Brown JQ, Heide RSV (2020) Pulmonary and cardiac pathology in Covid-19: the first autopsy series from new orleans. medRxiv. https://doi.org/10.1101/2020.04.06.20050575 Published online April 10, 2020

18. Escher R, Breakey N, Lammle B (2020) Severe COVID-19 infection associated with endothelial activation. Thromb Res 190:62

19. Wu C, Chen X, Cai Y, Xia J, Zhou X, Xu S et al (2020) Risk factors associated with acute respiratory distress syndrome and death in patients with coronavirus disease 2019 pneumonia in Wuhan, China. JAMA Intern Med 180(7):934-943

20. Guan W, Ni Z, Hu Y, Liang W, Ou C, He J et al (2020) Clinical characteristics of coronavirus disease 2019 in China. N Engl J Med 382(18):1708-1720

21. Huang C, Wang Y, Li X, Ren L, Zhao J, Hu Y et al (2020) Clinical features of patients infected with 2019 novel coronavirus in Wuhan, China. Lancet 395(10223):497-506

22. Taha M, Samavati L (2021) Antiphospholipid antibodies in COVID-19: a meta-analysis and systematic review. RMD Open 2021(7):e001580. https://doi.org/10.1136/rmdopen-2021-001580

23. https://www.hematology.org/covid-19/covid-19-and-apl-ab. Accessed 7 Dec 2020

24. Pineton de Chambrun M, Frere C, Amoura Z, Martin-Toutain I, Mathian A et al (2020) High frequency of antiphospholipid antibodies in critically ill COVID-19 patients: a link with hypercoagulability? J Intern Med. https:// doi.org/10.1111/joim.13126

25. Amezcua-Guerra LM, Rojas-Velasco G, Brianza-Padilla M, Vázquez-Rangel A, Márquez-Velasco R, Baranda-Tovar F et al (2020) Presence of antiphospholipid antibodies in COVID-19: Case series study. Ann Rheum Dis. https://doi.org/10.1136/annrheumdis-2020-218100

26. Xiao M, Zhang Y, Zhang S, Qin X, Xia P, Cao W et al (2020) Antiphospholipid antibodies in critically ill patients with COVID-19. Arthritis Rheum 72:1998-1994

27. Zhang Y, Cao W, Jiang W, Xiao M, Li Y, Tang N et al (2020) Profile of natural anticoagulant, coagulant factor and anti-phospholipid antibody in critically ill COVID-19 patients. J Thromb Thrombolysis 50:580-586

28. Le Joncour A, Frere C, Martin-Toutain I, Gougis P, Ghillani-Dalbin P, Maalouf $G$ et al (2021) Antiphospholipid antibodies and thrombotic events in COVID-19 patients hospitalized in medicine ward. Autoimmun Rev 20(2):102719
29. Castillo-Martinez D, Torres Z, Amezcua-Guerra LM, Pineda C (2021) Are antiphospholipid antibodies just a common epiphenomenon or are they causative of immune-mediated coagulopathy in COVID-19? Clin Rheumatol 40:3015-3019

30. Pengo V, Ruffatti A, Legnani C, Testa S, Fierro T, Marongiu F et al (2011) Incidence of a first thromboembolic event in asymptomatic carriers of high-risk antiphospholipid antibody profile: a multicenter prospective study. Blood 118:4714-4718

31. Pengo V, Biasiolo A, Bison E, Chantarangkul V, Tripodi A, Italian Federation of Anticoagulant Clinics (FCSA) (2007) Antiphospholipid antibody ELISAs: survey on the performance of clinical laboratories assessed by using lyophilized affinity-purifed IgG with anticardiolipin and anti-beta2glycoprotein I activity. Thromb Res 120:127-133

32. Pengo V, Tripodi A, Reber G, Rand JH, Ortel TL, Galli M et al (2009) Subcommittee on Lupus Anticoagulant/Antiphospholipid Antibody of the Standardization Committee of the International Society on Haemostasis, Update of the guidelines for lupus anticoagulant detection. Subcommittee on Lupus Anticoagulant/Antiphospholipid Antibodies of the scientific and standardization committee of the international society on thrombosis and haemostasis. J Thromb Haemost 7:1737-1740

33. de Groot PG, Lutters B, Derksen RHWM, Lisman T, Meijers JCM, Rosendaal FR (2005) Lupus anticoagulants and the risk of first episode of deep venous thrombosis. J Thromb Haemost 3:1993-1997

34. Pericleous C, Ferreira I, Borghi O, Pregnolato F, McDonnell T, Garza-Garcia A et al (2016) Measuring IgA anti-beta2-glycoprotein I and lgG/lgA anti-domain I antibodies adds value to current serological assays for the antiphospholipid syndrome. PLoS ONE. https://doi.org/10.1371/journal. pone. 0156407

35. Kungwankiattichai S, Nakkinkun Y, Owattanapanich W, Ruchutrakool T (2020) High incidence of antiphospholipid antibodies in newly diagnosed patients with lymphoma and a proposed aPL predictive score. Clin Appl Thromb Hemost 26:107602962092839

36. Devreese KMJ, Ortel TL, Pengo V, de Laat B (2018) for the subcommittee on lupus anticoagulant/antiphospholipid antibodies: laboratory criteria for antiphospholipid syndrome: communication from the SSC of the ISTH. J Thromb Haemost 16:809-813

37. Vassalo J, Spector N, Ede M, Soares M, Salluh JIF (2014) Antiphospholipid antibodies in critically ill patients. Rev Bras Ter Intensiva 26(2):176-182

38. Wong R, Favaloro E, Pollock W, Wilson R, Hendle M, Adelstein S et al (2004) A multi-center evaluation of the intra-assay and inter-assay variation of commercial and in-house anti-cardiolipin antibody assays. Pathology 36(2):182-192

39. Vanoverschelde L, Kelchtermans H, Musial J, de Laat B, Devreese KMJ (2019) Influence of anticardiolipin and anti-ß2 glycoprotein I antibody cut of values on antiphospholipid syndrome classification. Res Pract Thromb Haemost 3:515-527

\section{Publisher's Note}

Springer Nature remains neutral with regard to jurisdictional claims in published maps and institutional affiliations.

\section{Submit your manuscript to a SpringerOpen ${ }^{\circ}$ journal and benefit from:}

- Convenient online submission

- Rigorous peer review

- Open access: articles freely available online

- High visibility within the field

- Retaining the copyright to your article

Submit your next manuscript at $\boldsymbol{\nabla}$ springeropen.com 